\title{
Evaluación del grado de concordancia entre los resultados del examen histopatológico y del cultivo bacteriológico en el diagnóstico de tuberculosis bovina en México
}

Jacobo Carrisoza Urbina ${ }^{a+}$

0000-0002-8424-930X

Estela Flores Velázquez ${ }^{\mathrm{b}}$

0000-0003-2738-7658

José A. Gutiérrez Reyes ${ }^{b}$

Noé Orlando Juárez López a*

0000-0002-4710-702X

a Departamento de Genética y Bioestadística Facultad de Medicina Veterinaria y Zootecnia

Universidad Nacional Autónoma de México

Av. Universidad 3000, 04510, DF, México

${ }^{\mathrm{b}}$ Dirección General de Salud Animal

Servicio Nacional de Sanidad, Inocuidad

y Calidad Agroalimentaria

Av. Municipio Libre 377, Piso 7 Ala B,

Col. Santa Cruz Atoyac, 03310, DF, México

+ Este estudio es la tesis

de licenciatura del primer autor.

*Autor para correspondencia:

Tel: + 52 55-5622-5894

Correo electrónico:

noeorlando@comunidad.unam.mx

Recibido: 2015-01-23

Aceptado: $2015-09-28$

Publicado: $2015-09-30$

Información y declaraciones adicionales en la página 10

(a) Derechos de autor: Jacobo Carrisoza Urbina et al. 2015
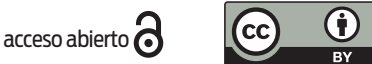

Distribuido bajo una Licencia Creative Commons

Atribución 4.0 Internacional (CC-BY 4.0)

\section{Resumen}

La tuberculosis bovina es una enfermedad compleja, difícil de diagnosticar, controlar y erradicar y tiene un impacto negativo en el sector ganadero. El objetivo de este estudio fue evaluar el grado de concordancia entre los resultados del examen histopatológico y del cultivo bacteriológico en el diagnóstico de lesiones de tuberculosis bovina realizado en los laboratorios certificados por la Secretaría de Agricultura, Ganadería, Desarrollo Rural, Pesca y Alimentación (SAGARPA) a nivel nacional, en el periodo comprendido entre enero de 2009 y diciembre de 2012 en México.

La información se obtuvo de 10,818 muestras de bovinos de matanza regular, a las cuales no se los sometió a la prueba de tuberculina, pero tenían lesiones que sugerían tuberculosis. Esas muestras se enviaron a diez laboratorios autorizados. Mediante el coeficiente kappa de Cohen, se midió la confiabilidad del diagnóstico; se obtuvo una concordancia general entre los resultados del examen histopatológico y del cultivo bacteriológico de $k=0.633$, con un intervalo de confianza (IC) del 95\% (0.618-0.649), lo cual indica una concordancia buena entre las dos pruebas a nivel nacional.

El laboratorio de Chihuahua obtuvo el valor más alto de kappa [ $k=0.784$; IC del 95\% (0.754-0.814)] y el laboratorio de la Región Lagunera en el estado de Coahuila obtuvo un valor bajo de concordancia $[k=0.334$; IC del 95\% (0.257-0.412)]. El número de muestras positivas en el cultivo bacteriológico fue bajo en Tamaulipas (21 muestras), Sonora (41 muestras) y Yucatán (45 muestras), ya que la prevalencia de tuberculosis en esos Estados es $\leq$ 0.04\%.

El grado de discordancia en algunos laboratorios involucra factores como la falta de recursos económicos, de infraestructura y de capacitación. El procedimiento correcto para la toma, conservación, envío y procesamiento de las muestras es crucial para optimizar los resultados bacteriológicos e histopatológicos y, así, llegar al correcto diagnóstico de la tuberculosis bovina y de diagnósticos diferenciales.

Palabras clave: Tuberculosis bovina; Concordancia; Histopatología; Bacteriología; Kappa de Cohen. 


\section{Introducción}

La tuberculosis bovina provoca pérdidas económicas importantes para los ganaderos. Se estima que más de 50 millones de bovinos están infectados en todo el mundo, lo que genera un egreso aproximado de $\$ 3,000$ millones de dólares anuales, debido a los gastos asociados con las pruebas de diagnóstico, el sacrificio de los animales infectados, la pérdida de acuerdos comerciales, las restricciones en la movilización, el pago de indemnización, el mantenimiento de los programas de control de la enfermedad, tanto regionales como federales, y la investigación para el desarrollo de mejores estrategias de control (Waters et al., 2012).

México exporta 1.2 millones de novillos a Estados Unidos de América, lo que se traduce en un intercambio comercial por más de 480 millones de dólares (SAGARPA, 2013) y la tuberculosis bovina amenaza con restringir este comercio. Además, 28\% de la leche producida a nivel nacional se consume cruda o se transforma sin pasteurización en derivados lácteos, lo que implica un alto riesgo zoosanitario y de salud pública (CANATB, 2004; Hernández, 1998).

La tuberculosis bovina es una enfermedad compleja y crónica, difícil de diagnosticar, controlar y erradicar, además tiene un impacto negativo en la industria ganadera. El agente etiológico es Mycobacterium bovis, que afecta a una amplia gama de mamíferos, incluyendo los seres humanos (O'Reilly, Daborn, 1995; Acha, 2003).

Con el objetivo de prevenir, controlar y erradicar esta enfermedad, México ha establecido la Campaña Nacional contra la Tuberculosis Bovina (Mycobacterium bovis), sustentada en la Norma Oficial Mexicana NOM-031-ZOO-1995. Esta norma establece que las muestras con lesiones que sugieran tuberculosis, identificadas en la matanza regular de bovinos que reaccionaron a la prueba de tuberculina, deben recolectarse y enviarse a los laboratorios autorizados por la SAGARPA (Secretaría de Agricultura, Ganadería, Desarrollo Rural, Pesca y Alimentación), para el diagnóstico de la tuberculosis bovina a través de pruebas histopatológicas y bacteriológicas.

Después de aislar e identificar $M$. bovis, el laboratorio notifica a la Dirección General de Salud Animal del Servicio Nacional de Sanidad, Inocuidad y Calidad Agroalimentaria (SENASICA), quien utiliza diferentes estrategias para ubicar el hato de origen infectado y controlar la enfermedad.

Los exámenes histopatológicos y el cultivo bacteriológico son los métodos más confiables para la detección de micobacterias en animales en quienes se sospecha infección por M. bovis y otras micobacteriosis. Sin embargo, la sensibilidad y la especificidad de estas pruebas se ve afectada por varios factores, que van desde la correcta toma de la muestra, el medio de transporte utilizado, el tiempo de procesamiento de la muestra, los métodos de desinfección de la muestra para el cultivo bacteriológico, la cantidad de micobacterias viables y el control de micobacterias por el sistema inmune del huésped, entre otros (Albernaz et al., 2014.; Pinto et al., 2002).

La confirmación de la enfermedad por medio del examen histopatológico y el cultivo bacteriológico es invaluable para el Sistema de Vigilancia Epidemiológica de la Tuberculosis Bovina (Kaneenea et al., 2006; Frankena et al., 2007; Shittu et al., 2013). En este contexto, el grado de concordancia entre el examen histopatológico y el cultivo bacteriológico es de suma importancia, especialmente cuando éstos determinan el diagnóstico de una enfermedad de notificación obligatoria, como es el caso de la tuberculosis bovina (OIE).

El coeficiente kappa de Cohen se utiliza para medir la concordancia entre pruebas; indica la asociación entre variables categóricas y binarias. Es una estima- 
ción del grado de asociación que va más allá del azar. Esta prueba estadística se ha utilizado en el campo médico para conocer la confiabilidad y la validez de un diagnóstico y para estimar el factor de riesgo entre pruebas (Kraemer et al., 2002).

Los objetivos de este estudio, cuyo periodo comprende de enero de 2009 a diciembre de 2012, fueron: 1) evaluar el grado de concordancia entre los resultados del examen histopatológico y del cultivo bacteriológico de lesiones que sugieren tuberculosis, donde los inspectores localizaron las lesiones durante el sacrificio rutinario de bovinos en el rastro y las muestras se enviaron a los laboratorios autorizados por la SAGARPA para el diagnóstico de tuberculosis bovina, y 2) determinar si el grado de concordancia se mantuvo constante durante esos años.

\section{Material y métodos}

Datos

La Dirección de Campañas Zoosanitarias del SENASICA recopila información de los resultados obtenidos del examen histopatológico y de cultivo bacteriológico proporcionados por los laboratorios autorizados por la SAGARPA, localizados en Chiapas, Chihuahua, Jalisco, la Región Lagunera, Monterrey, Sonora, Tabasco, Tamaulipas, Veracruz y Yucatán. Estos laboratorios están autorizados para realizar el diagnóstico formal de tuberculosis bovina y manejan una elevada cantidad de muestras.

La base de datos analizada consistió de los resultados de 10,818 muestras de tejido procedentes de la matanza regular de bovinos de engorda, lecheros y de doble propósito que presentaron lesiones sugestivas a tuberculosis en el periodo de enero de 2009 a diciembre de 2012.

\section{Muestras de tejido}

Las muestras de tejido para los estudios de histopatología y bacteriología se tomaron en los órganos con lesiones o secreciones compatibles, o que sugerían tuberculosis, de acuerdo con la norma NOM-03 1-ZOO-1995 y la guía epidemiológica de 2015 (SENASICA), de la siguiente manera:

a) Nódulos linfáticos: se toman muestras de los nódulos linfáticos de la cabeza, pre-escapulares, mediastínicos anterior y posterior, y los bronquiales derecho e izquierdo. Los nódulos linfáticos mesentéricos se muestrean en caso de tuberculosis miliar.

b) Pulmones: se toman muestras de lesiones caseosas y calcificadas. La muestra debe incluir $2 \mathrm{~cm}$ de tejido alrededor de la lesión.

c) Útero: debe tomarse muestra del órgano y del exudado (pus, secreción amarilla con aspecto de leche cuajada).

d) Otros tejidos: bazo, hígado, riñón, médula ósea, ovarios, testículos y glándula mamaria.

\section{Diagnóstico histopatológico}

El diagnóstico histopatológico es auxiliar en la detección de la tuberculosis cuando se observan lesiones macroscópicas o microscópicas en una muestra de tejido. Se 
tomaron muestras de tejido de $2 \mathrm{~cm}^{3}$ sin tejido adiposo; se fijaron en formalina y se incluyeron en parafina para obtener los cortes histológicos que se tiñeron con hematoxilina-eosina y se observaron a través de un microscopio óptico. Los resultados fueron:

1. Negativo a tuberculosis: no se observaron lesiones granulomatosas típicas ni micobacterias.

2. Sugiere tuberculosis: se observaron granulomas típicos, pero no se detectaron bacterias ácido-alcohol resistentes.

\section{Diagnóstico bacteriológico}

Las muestras de tejido de $2 \mathrm{~cm}^{3}$ sin tejido adiposo se sumergieron en una solución saturada de borato de sodio, y se examinaron mediante microscopía y cultivo bacteriano. Los tejidos se homogeneizaron, se descontaminaron y se inocularon en los medios Stonebrink y Lowenstein-Jensen. Los tubos inoculados se incubaron a $37{ }^{\circ} \mathrm{C}$ durante 9 semanas; se registró cada semana el tiempo de crecimiento de las bacterias y la formación de pigmento. En general, las bacterias atípicas crecieron en 7-21 días, mientras que Mycobacterium bovis creció después de la cuarta semana de incubación. Las pruebas de histopatología y cultivo bacteriano se realizaron al mismo tiempo.

\section{Análisis estadístico}

Se utilizó la prueba estadística kappa de Cohen para evaluar la concordancia entre los resultados del examen histopatológico y del cultivo bacteriológico, sin suponer que una de estas dos pruebas de diagnóstico fuera mejor que la otra. También se calcularon los valores mínimos y máximos, según lo sugerido por Lantz et al. (1996), debido a que la prevalencia de la característica en estudio puede afectar el valor de kappa. La técnica de Jackknife se utilizó para calcular el intervalo de confianza de kappa (Efron, Tibshirani, 1993).

Mediante la prueba de homogeneidad de kappas, se compararon los índices de kappa a lo largo de los años para determinar si estos índices variaban entre años $(\alpha=0.05)$. El estadístico de kappa tiene una distribución Ji-cuadrada y la hipótesis nula $\left(\mathrm{H}_{0}\right)$ a probar indica que todos los índices kappa que se comparan son iguales.

La concordancia se analizó con el programa EPIDAT 4.0 (OPS-OMS, 2014).

\section{Resultados y discusión}

El cuadro 1 contiene el número total de muestras enviadas a los laboratorios autorizados por la SAGARPA para el diagnóstico de $M$. bovis. Los resultados de ambas pruebas diagnósticas provenientes de los diez laboratorios certificados se muestran en el cuadro 2.

Los estados con la frecuencia de envíos más alta fueron Jalisco, Chihuahua y Veracruz (cuadro 1), porque, junto con Chiapas, tienen la población más grande de ganado bovino en el país. Sin embargo, Veracruz, con una población de 
Cuadro 1. Muestras totales de la matanza regular recibidas por los laboratorios certificados de 2009 a 2012.

\begin{tabular}{|c|c|c|c|c|c|}
\hline Laboratorio & $\mathbf{2 0 0 9}$ & $\mathbf{2 0 1 0}$ & $\mathbf{2 0 1 1}$ & $\mathbf{2 0 1 2}$ & Total \\
\hline Chiapas & 215 & 187 & 270 & 193 & 865 \\
\hline Chihuahua & 553 & 325 & 625 & 562 & 2065 \\
\hline Jalisco & 557 & 746 & 844 & 413 & 2560 \\
\hline Región Lagunera & 93 & 91 & 61 & 84 & 329 \\
\hline Monterrey & 29 & 235 & 364 & 326 & 954 \\
\hline Sonora & 251 & 262 & 92 & 194 & 799 \\
\hline Tabasco & 188 & 150 & 324 & 156 & 818 \\
\hline Tamaulipas & 91 & 74 & 39 & 131 & 335 \\
\hline Veracruz & 364 & 236 & 519 & 419 & 1538 \\
\hline Yucatán & 87 & 91 & 217 & 160 & 555 \\
\hline Total & 2,428 & 2,397 & 3,355 & 2,638 & 10,818 \\
\hline
\end{tabular}

3.7 millones de bovinos, recibió menos muestras que Jalisco (cuadro 1), que tiene 3 millones de bovinos (SIAP). Estas cifras, probablemente, se relacionan con que Jalisco tiene el 10.6\% del total de ganado lechero del país, con una prevalencia de tuberculosis bovina más alta (0.067-0.25\%) que Veracruz (0.003-0.046\%), el cual tiene solamente el $1.5 \%$ de la población total de ganado lechero (SAGARPA).

Con los porcentajes de los resultados positivos para tuberculosis a través de, o histopatología, o cultivo bacteriológico, o ambas pruebas de los cuadros 1 y 2, se calcularon los porcentajes del cuadro 3. Por cada 100 muestras recibidas, 39 fueron positivas tanto por histopatología como por bacteriología en el laboratorio de Jalisco y solamente 22 en el laboratorio de Veracruz (cuadro 3).

Asimismo, dos estados con aproximadamente el mismo número de bovinos (1.5 millones), Tabasco y Tamaulipas, mostraron resultados muy diferentes. El laboratorio de Tabasco recibió más del doble de muestras que el de Tamaulipas; $12 \%$ de las muestras dieron positivo para tuberculosis en Tabasco y 3\%, para Tamaulipas (cuadro 3). Tabasco tiene una prevalencia de tuberculosis de 0.05-0.36\% y Tamaulipas de 0.038\% (SAGARPA).

Por la prueba de cultivo bacteriológico, se identificó M. bovis en 2,499 muestras $(206+2,293$; cuadro 2) de 10,818 muestras sospechosas, lo que equivale al $23.1 \%$ del total nacional (cuadro 3). Estos resultados concuerdan con lo encontrado en Brasil (Araújo et al., 2005), donde de un total de 72 muestras, 23.6\% fueron positivas por cultivo bacteriológico. En Ecuador, 36.3\% de las muestras que sugirieron tuberculosis resultaron positivas después del cultivo bacteriológico (Pérez, 2011).

Los laboratorios con la mayor cantidad de resultados positivos a través del cultivo bacteriológico fueron Jalisco con 41.2\%, y la Región Lagunera con 29.5\% (cuadro 3). Jalisco y Coahuila tienen 329, 189 y 438,048 bovinos lecheros, respectivamente (SIAP), la población más grande en el país, y la prevalencia de tuberculosis es de $0.03-0.46 \%$ para Jalisco y 0.07->4\% para la Región Lagunera (SAGARPA). Pérez et al. (2008) indican que la prevalencia de tuberculosis en los bovinos lecheros puede alcanzar el 16\%.

Por otra parte, los laboratorios con el índice más bajo de resultados positivos fueron Sonora con 5.1\% y Tamaulipas con 6.3\% (cuadro 3). Estos estados son 
Cuadro 2. Resultados de las pruebas de histopatología (H) y bacteriología (B)

proporcionados por los laboratorios certificados, de 2009 a 2012.

\begin{tabular}{|c|c|c|c|c|c|c|c|c|c|c|c|c|c|c|c|c|c|c|c|c|}
\hline & \multicolumn{4}{|c|}{2009} & \multicolumn{4}{|c|}{2010} & \multicolumn{4}{|c|}{2011} & \multicolumn{4}{|c|}{2012} & \multicolumn{4}{|c|}{ Total } \\
\hline & $H / B$ & $H / B$ & $H / B$ & $H / B$ & $H / B$ & $H / B$ & $H / B$ & $H / B$ & $H / B$ & $H / B$ & $H / B$ & $H / B$ & $H / B$ & $H / B$ & $H / B$ & $H / B$ & $H / B$ & $H / B$ & $H / B$ & $H / B$ \\
\hline Laboratorio & $-/-$ & $-/+$ & $+/-$ & $+/+$ & $-/-$ & $-/+$ & $+/-$ & $+/+$ & $-1-$ & $-/+$ & $+/-$ & $+/+$ & $-/-$ & $-/+$ & $+/-$ & $+/+$ & $-/-$ & $-/+$ & $+/-$ & $+/+$ \\
\hline Chiapas & 135 & 0 & 39 & 41 & 100 & 0 & 35 & 52 & 108 & 7 & 74 & 81 & 109 & 12 & 23 & 49 & 452 & 19 & 171 & 223 \\
\hline Chihuahua & 379 & 6 & 29 & 139 & 235 & 0 & 25 & 65 & 426 & 3 & 68 & 128 & 409 & 5 & 43 & 105 & 1,449 & 14 & 165 & 437 \\
\hline Jalisco & 188 & 5 & 112 & 252 & 292 & 30 & 136 & 288 & 318 & 7 & 182 & 337 & 163 & 2 & 115 & 133 & 961 & 44 & 545 & 1,010 \\
\hline Región Lagunera & 4 & 3 & 61 & 25 & 33 & 2 & 36 & 20 & 25 & 1 & 10 & 25 & 57 & 1 & 6 & 20 & 119 & 7 & 113 & 90 \\
\hline Monterrey & 0 & 3 & 19 & 7 & 221 & 12 & 2 & 0 & 325 & 7 & 20 & 12 & 299 & 3 & 10 & 14 & 845 & 25 & 51 & 33 \\
\hline Sonora & 227 & 4 & 8 & 12 & 254 & 5 & 2 & 1 & 83 & 6 & 0 & 3 & 179 & 8 & 5 & 2 & 743 & 23 & 15 & 18 \\
\hline Tabasco & 84 & 6 & 60 & 38 & 89 & 17 & 12 & 32 & 269 & 17 & 12 & 26 & 142 & 3 & 9 & 2 & 584 & 43 & 93 & 98 \\
\hline Tamaulipas & 90 & 0 & 1 & 0 & 61 & 1 & 9 & 3 & 31 & 4 & 3 & 1 & 106 & 6 & 13 & 6 & 288 & 11 & 26 & 10 \\
\hline Veracruz & 206 & 1 & 40 & 117 & 117 & 1 & 45 & 73 & 348 & 3 & 68 & 100 & 316 & 4 & 49 & 50 & 987 & 9 & 202 & 340 \\
\hline Yucatán & 70 & 1 & 14 & 2 & 77 & 1 & 10 & 3 & 161 & 1 & 33 & 22 & 138 & 8 & 7 & 7 & 446 & 11 & 64 & 34 \\
\hline Nacional & 1,383 & 29 & 383 & 633 & 1,479 & 69 & 312 & 537 & 2,094 & 56 & 470 & 735 & 1,918 & 52 & 280 & 388 & 6,874 & 206 & 1,445 & 2,293 \\
\hline
\end{tabular}

Resultado de la prueba: (-) negativo, (+) positivo.

Cuadro 3. Porcentaje de muestras que dieron positivo para tuberculosis por histopatología, cultivo bacteriológico y por ambas pruebas de 2009 a 2012.

\begin{tabular}{|c|c|c|c|c|c|c|c|}
\hline Laboratorio & $\begin{array}{l}\text { Muestras } \\
\text { recibidas }\end{array}$ & $\begin{array}{l}\text { Muestras positivas } \\
\text { por histopatologia }\end{array}$ & $\%$ & $\begin{array}{l}\text { Muestras positivas } \\
\text { por cultivo bacteriológico }\end{array}$ & $\%$ & $\begin{array}{l}\text { Muestras positivas } \\
\text { por ambas pruebas }\end{array}$ & $\%$ \\
\hline Chiapas & 865 & 394 & 45.5 & 242 & 28.0 & 223 & 25.8 \\
\hline Chihuahua & 2,065 & 602 & 29.1 & 451 & 21.8 & 437 & 21.2 \\
\hline Jalisco & 2,560 & 1,555 & 60.7 & 1,054 & 41.2 & 1,010 & 39.4 \\
\hline Región Lagunera & 329 & 203 & 61.7 & 97 & 29.5 & 90 & 27.4 \\
\hline Monterrey & 954 & 84 & 8.8 & 58 & 6.1 & 33 & 3.5 \\
\hline Sonora & 799 & 33 & 4.1 & 41 & 5.1 & 18 & 2.2 \\
\hline Tabasco & 818 & 191 & 23.3 & 141 & 17.2 & 98 & 12.0 \\
\hline Tamaulipas & 335 & 36 & 10.7 & 21 & 6.3 & 10 & 3.0 \\
\hline Veracruz & 1,538 & 542 & 35.2 & 349 & 22.7 & 340 & 22.1 \\
\hline Yucatán & 555 & 98 & 17.7 & 45 & 8.1 & 34 & 6.1 \\
\hline Total & 10,818 & 3,738 & 34.5 & 2,499 & 23.1 & 2,293 & 21.2 \\
\hline
\end{tabular}


importantes por su producción de bovinos de engorda, en los que la prevalencia de tuberculosis es de $0.04 \%$ (SAGARPA).

En el examen histopatológico, 34.5\% de las muestras dieron positivo para tuberculosis; el valor mínimo fue de $4.1 \%$ para el laboratorio en Sonora y el máximo fue de 61.7\% para la Región Lagunera (cuadro 3). En Argentina en el mismo examen, Latini et al. (1997) encontraron que 81.8\% de 248 muestras dieron positivo para tuberculosis en el examen histopatológico. Sin embargo, Pinto et al. (2002) reportaron una baja sensibilidad en este estudio: 38.7\%. Latini et al. (1997) indican que la sensibilidad de la prueba de histopatología se afecta por la técnica usada, la cantidad de laminillas examinadas, la manera en la que se colectan las muestras, y cómo se envían y preservan.

La cuantificación del grado de concordancia entre el examen histopatológico y el cultivo bacteriológico permite ver, de manera general, el grado de concordancia entre estas dos pruebas para detectar tuberculosis. Bajos valores de kappa indican discrepancia entre las pruebas, probablemente debido a la detección de la infección a través de diferentes mecanismos que podrían interpretarse como resultados falsos positivos o negativos, mientras que valores altos de kappa indican precisión en el diagnóstico.

Estrada-Chavez et al. (2004) mencionan que los resultados falsos positivos generan problemas sociales y económicos, porque un resultado equivocado puede contribuir a la diseminación de la enfermedad.

Los resultados de la concordancia generalmente se clasifican según la siguiente escala (Gilchrist, 2009):

a) -1 a 0.00 , concordancia totalmente inadecuada

b) 0.01 a 0.39 , concordancia insuficiente

c) 0.40 a 0.75 , buena concordancia

d) 0.76 a 0.99 , excelente concordancia

e) 1, concordancia perfecta

El estadístico kappa de Cohen a nivel nacional indica que la concordancia entre el examen histopatológico y el cultivo bacteriológico de lesiones que sugieren tuberculosis fue $\mathrm{k}=0.634$ (buena concordancia), con un intervalo de confianza del 95\%, es decir, entre 0.618 y 0.650 (cuadro 4). Este valor fue mayor que el obtenido en Ecuador, $k=0.49$ (Pérez et al., 2011 ) y Argentina, $k=0.48$ (Latini et al., 1997).

Sin embargo, los estados variaron en sus estadísticos kappa de Cohen. El laboratorio de Chihuahua presentó una concordancia excelente con un coeficiente $k=0.784$, mientras que la concordancia fue insuficiente en dos laboratorios, el de la Región Lagunera, donde $\mathrm{k}=0.334$, y el de Tamaulipas con un coeficiente $k=0.295$. El resto de los laboratorios presentó una buena concordancia.

La diferencia de valores entre los laboratorios podría deberse a la manipulación de la muestra al momento del envió, el tiempo entre el muestreo y el procesamiento de muestras, la baja cantidad de micobacterias viable, la falta de recursos económicos, la infraestructura inadecuada y las deficiencias en la capacitación del personal de laboratorio. Sin embargo, como se mencionó, la prueba de histopatología puede no ser confiable, tal como Pinto et al. (2002) reportaron en el 38.7\% de sus resultados positivos. 
Cuadro 4. Kappa con un intervalo de 95\% de confianza, y valores mínimos y máximos de kappa.

\begin{tabular}{|c|c|c|c|c|c|}
\hline \multicolumn{7}{|c|}{ Intervalo de confianza al 95\% } \\
\hline Laboratorio & $\begin{array}{c}\text { kappa } \\
\text { general }\end{array}$ & $\begin{array}{c}\text { Límite } \\
\text { inferior }\end{array}$ & $\begin{array}{c}\text { Límite } \\
\text { superior }\end{array}$ & $\begin{array}{c}\text { kappa } \\
\text { mínimo }\end{array}$ & $\begin{array}{c}\text { kappa } \\
\text { máximo }\end{array}$ \\
\hline Chiapas & 0.543 & 0.489 & 0.596 & -0.123 & 0.581 \\
\hline Chihuahua & 0.784 & 0.754 & 0.814 & -0.045 & 0.828 \\
\hline Jalisco & 0.556 & 0.527 & 0.585 & -0.128 & 0.563 \\
\hline Región Lagunera & 0.334 & 0.257 & 0.412 & -0.213 & 0.356 \\
\hline Monterrey & 0.423 & 0.317 & 0.530 & -0.042 & 0.842 \\
\hline Sonora & 0.462 & 0.317 & 0.606 & -0.024 & 0.905 \\
\hline Tabasco & 0.489 & 0.416 & 0.562 & -0.091 & 0.676 \\
\hline Tamaulipas & 0.295 & 0.129 & 0.461 & -0.059 & 0.782 \\
\hline Veracruz & 0.672 & 0.633 & 0.712 & -0.074 & 0.731 \\
\hline Yucatán & 0.410 & 0.305 & 0.515 & -0.073 & 0.735 \\
\hline Nacional & 0.634 & 0.618 & 0.650 & -0.083 & 0.702 \\
\hline
\end{tabular}

Por otro lado, la baja prevalencia de tuberculosis $(<0.1-<0.01 \%)$ en 25 regiones podría también explicar la baja concordancia obtenida en algunos laboratorios. Si la prevalencia es baja, entonces el número de resultados verdaderamente "negativos" será alto, como también lo será la probabilidad de que tanto la histopatología como el cultivo bacteriológico diagnostiquen las muestras como "negativas" (USDA, 2009). Debido a que la estadística de kappa elimina la influencia del azar, se obtendrán valores de kappa bajos (0.115). Por ello, en el cuadro 4 se incluyeron los valores mínimos y máximos que ayudan al análisis de los datos (Lantz et al., 1996).

Diagnósticos diferenciales también deberían considerarse. Por ejemplo, Valencia et al. (1997) analizaron 505 muestras, de las cuales 49\% dio positivo para Mycobacterium; el resto diagnosticaron actinobacilosis, actinomicosis, granuloma eosinofílico y otras enfermedades.

Chakravorty et al. (2005) mencionan que el diagnóstico de tuberculosis cambia por: 1) la cantidad y volumen de muestra inadecuados, 2) el uso de la muestra en varias pruebas diagnósticas (histología/citología, análisis bioquímicos, microbiología y PCR) que puede acarrear una mala distribución de los microorganismos, 3) la naturaleza paucibacilar de las muestras, 4) la presencia de inhibidores que debilitan el rendimiento de técnicas basadas en la amplificación de ácido nucleico, y 5) técnicas de procesamiento de la muestra ineficientes en todo el mundo.

Shitaye et al. (2006) mencionan que los resultados falsos negativos se pueden asociar con una baja cantidad de micobacterias, que no está distribuida de manera uniforme a través de todo el cuerpo, en particular en muestras infectadas con M. bovis.

El cuadro 5 presenta los coeficientes de concordancia del periodo 2009 a 2012 de cada laboratorio. Existe suficiente evidencia estadística para inferir que el promedio general de los coeficientes de kappa ha permanecido constante a lo largo de los años, al igual que los coeficientes de kappa para los laboratorios de Chiapas, Jalisco y Tamaulipas ( $\mathrm{P}>0.05)$. Esto no es así para los otros laboratorios; los de Chihuahua, Jalisco y Veracruz tuvieron un coeficiente de concordancia más alto en 
Cuadro 5. Kappa \pm error estándar (EE) calculado para el periodo 2009 a 2012.

\begin{tabular}{|c|c|c|c|c|c|}
\hline & 2009 & 2010 & 2011 & 2012 & \\
\hline Laboratorio & Kappa \pm EE & Kappa \pm EE & Kappa \pm EE & Kappa \pm EE & $\boldsymbol{P}$ \\
\hline Chiapas & $0.569 \pm 0.05$ & $0.614 \pm 0.05$ & $0.559 \pm 0.05$ & $0.600 \pm 0.06$ & 0.8795 \\
\hline Chihuahua & $0.844 \pm 0.02$ & $0.790 \pm 0.03$ & $0.710 \pm 0.03$ & $0.760 \pm 0.03$ & $0.0072^{*}$ \\
\hline Jalisco & $0.590 \pm 0.03$ & $0.564 \pm 0.02$ & $0.570 \pm 0.02$ & $0.470 \pm 0.03$ & 0.0629 \\
\hline Región Lagunera & $-0.029 \pm 0.00$ & $0.254 \pm 0.00$ & $0.647 \pm 0.09$ & $0.794 \pm 0.07$ & 0* \\
\hline Monterrey & $-0.218 \pm 0.12$ & $-0.015 \pm 0.00$ & $0.433 \pm 0.09$ & $0.662 \pm 0.08$ & $0^{*}$ \\
\hline Sonora & $0.641 \pm 0.09$ & $0.210 \pm 0.18$ & $0.474 \pm 0.17$ & $0.201 \pm 0.14$ & $0.0322^{*}$ \\
\hline Tabasco & $0.313 \pm 0.05$ & $0.549 \pm 0.07$ & $0.591 \pm 0.06$ & $0.215 \pm 0.14$ & $0.0025^{*}$ \\
\hline Tamaulipas & $t$ & $0.320 \pm 0.15$ & $0.122 \pm 0.19$ & $0.310 \pm 0.11$ & 0.4302 \\
\hline Veracruz & $0.763 \pm 0.03$ & $0.610 \pm 0.04$ & $0.653 \pm 0.03$ & $0.548 \pm 0.04$ & $0.0063^{*}$ \\
\hline Yucatán & $0.162 \pm 0.11$ & $0.306 \pm 0.14$ & $0.487 \pm 0.07$ & $0.162 \pm 0.11$ & $0.0272^{*}$ \\
\hline Nacional & $0.633 \pm 0.01$ & $0.629 \pm 0.01$ & $0.643 \pm 0.01$ & $0.625 \pm 0.01$ & 0.8607 \\
\hline
\end{tabular}

† El laboratorio no obtuvo ningún resultado positivo para bacteriología en ese año.

$* P \leq 0.05$ : evidencia estadística suficiente para rechazar la hipótesis nula de igualdad.

2009. El de Sonora fue muy inconsistente, mientras que 2010 y 2011 fueron los mejores años para los laboratorios de Tabasco y Yucatán.

Son muy interesantes los resultados de la Región Lagunera y de Monterrey: el peor año para La Laguna fue 2009, y 2010 para Monterrey. Sin embargo, ambos laboratorios mejoraron notablemente en 2011 y 2012; seguramente se tomaron decisiones y se hicieron ajustes importantes. Este tipo de análisis es muy útil como herramienta de gestión y de control de calidad.

En el Estado de México, en un estudio de tuberculosis bovina, que tuvo una alta prevalencia, Estrada-Chávez et al. (2004) obtuvieron una concordancia de $k=0.52$. Estos resultados señalan la utilidad del índice de concordancia para el diagnóstico definitivo de tuberculosis en bovinos con una alta prevalencia, así como para la evaluación de otras pruebas diagnósticas.

Las discrepancias en el grado de concordancia entre laboratorios podrían deberse a que el cultivo de $M$. bovis es la prueba más utilizada, pero consume mucho tiempo y requiere de un laboratorio con una infraestructura específica, equipo especializado y personal altamente calificado (Bermudez et al., 2010). Mientras las diferencias en los índices de kappa entre los laboratorios podrían haberse manifestado por las diferencias en la fiabilidad de ambos métodos en cada laboratorio en un periodo.

En un estudio realizado en el estado de Baja California, México, se analizaron 553 muestras de vacas de las que se sospechaba tuberculosis y 268 dieron positivo por histopatología. Esas 268 muestras se sometieron a: a) un cultivo bacteriológico, que dio como resultado 104 muestras positivas, y b) un análisis de PCR, con el cual 123 muestras fueron positivas: 70 fueron positivas por dos vías, PCR y cultivo, y 53 fueron positivas sólo vía PCR. Estos hallazgos hablan de la complejidad del diagnóstico de $M$. bovis y de la utilidad de revisar los métodos de laboratorio para la confirmación de tuberculosis bovina, a fin de incluir el PCR como método de diagnóstico. 


\section{Conclusiones}

Este estudio demostró que el índice de concordancia entre la prueba de histopatología y el cultivo bacteriológico para el diagnóstico de tuberculosis bovina a nivel nacional es bueno. También evidenció que el número de muestras positivas de acuerdo con el cultivo bacteriológico se relacionó con la prevalencia de tuberculosis bovina reportada por las agencias nacionales zoosanitarias. Sin embargo, existe una necesidad de mejorar los procedimientos asociados con el diagnóstico de esta enfermedad en algunos laboratorios certificados por la SAGARPA en México. Esta información puede ser útil como herramienta para la toma de decisiones en las instancias gubernamentales.

\section{Financiamiento}

Este estudio fue financiado por el Departamento de Genética y Bioestadística, Facultad de Medicina Veterinaria y Zootecnia.

\section{Agradecimientos}

Los autores desean agradecer al Departamento de Genética y Bioestadística la ayuda financiera concedida a Jacobo Carrisoza Urbina.

\section{Conflictos de interés}

Los autores declaran que no tienen conflictos de interés.

\section{Contribución de los autores}

Jacobo Carrisoza Urbina: recolectó los datos, interpretó los resultados y escribió el manuscrito.

Estela Flores Velásquez y José A. Gutiérrez Reyes: recolectaron los datos e interpretaron los resultados.

Noé O. Juárez López: diseñó el estudio, hizo el análisis estadístico, interpretó los resultados y escribió el manuscrito.

\section{Referencias}

1) Acha PN, Szifres B. 2003. Zoonosis y enfermedades transmisibles comunes al hombre y a los animales. $3^{a}$ ed. Washigton, DC: Organización Panamericana de la Salud.

2) Albernaz TT, Chavez CM, Limar HD, Silva SN, Cardoso PD, Lopez ATC, Brito FM, Silva BJ, Salvarani MF. 2015. Comparison of the tuberculin test, histopathological examination, and bacterial culture for the diagnosis of tuberculosis (Mycobacterium bovis) in buffaloes (Bubalus bubalis) in Brazil. Tropical Animal Health and Production, 47. DOI: 10.1007/s1 1250-015-0842-3. http://dx.doi. org/10.1007/s1 1250-015-0842-3

3) Araújo CP, Leite CQF, Prince KA, Jorge KSG, Osório ALAR. 2005. Mycobacterium bovis identification by molecular method from post-mortem inspected cattle obtained in abattoirs of Mato Grosso do Sul, Brazil. Memorias del Instituto Oswaldo Cruz, 100:749-752. 
4) Bermudez HR, Renteria ET, Medina BG. 2010. Correlation between histopathological, bacteriological and PCR diagnosis of bovine tuberculosis. Journal of Animal and Veterinary Advances, 9:2082-2084.

5) [CANATB] Campaña Nacional para el Control y la Erradicación de la Tuberculosis Bovina en México. 2004. Memorias de la Reunión Anual del Consejo Nacional de Salud Animal. México, DF.

6) Chakravorty S, Sen MK, Tyagi JS. 2005. Diagnosis of extrapulmonary tuberculosis by smear, culture, and PCR using universal sample processing technology. Journal of Clinical Microbiology, 43:4357-62.

7) Efron B, Tibshirani RJ. 1993. An introduction to the bootstrap. New York, Chapman \& Hall.

8) Estrada-Chávez C, Otero FD, Díaz CA, Villegas-Sepúlveda N, González RP, Salazar DG. 2004. Concordancia de la PCR y métodos rutinarios para el diagnóstico de tuberculosis bovina. Veterinaria México, 35:225-236.

9) Frankena K, White WP, O'Keeffe J, Costello E, Martin WS, Grevenhof IV. 2007. Quantification of the relative efficiency of factory surveillance in the disclosure of tuberculosis lesions in attested Irish cattle. Veterinary Record, 161:679-684.

10) Gilchrist MJ. 2009. Weighted $2 \times 2$ kappa coefficients: recommended indices of diagnostic accuracy for evidence-based practice. Journal of Clinical Epidemiology, 62:1045-1053.

11) Hernandez J, Baca D. 1998. Effect of tuberculosis on milk production in dairy cows. Journal of the American Veterinary Medical Association, 213(6):851-4.

12) Kaneenea JB, Millera AN, Meyerb R. 2006. Abattoir surveillance: The U.S. experience. Veterinary Microbiology, 112:273-282.

13) Kraemer $\mathrm{CH}$, Vyjeyanthi S, Periyakoil, Art Noda. 2002. Kappa coefficients in medical research. Statistics in Medicine, 21:2109-2129.

14) Lantz CA, Nebenzahl E. 1996. Behavior and interpretation of the k statistic: resolution of the two paradoxes. J Clin Epidemiol. 49(4):431-4.

15) Latini O, Canal AM, Ferrara ME, Sequeira MD, Bagnaroli RV, Torres P. 1997. Confiabilidad en la determinación de prevalencia de infección por Mycobacterium bovis en ganado bovino por decomisos en frigoríficos. Archivos de medicina veterinaria, 29(2): 197-204.

16) [NOM-031] Norma Oficial Mexicana [21 ago 1998]. NOM-031-ZOO-1995. Campaña Nacional contra la Tuberculosis Bovina (Mycobacterium bovis). DF, México: DOF- SAGARPA.

17) O'Reilly LM, Daborn CJ. 1995. The epidemiology of Mycobacterium bovis infections in animals and man. A review. Tubercle and Lung Disease, 76:1-46.

18) [OIE] World Organisation for Animal Health. Manual of Diagnostic Tests and Vaccines for Terrestrial Animals. 2014. http://www.oie.int/en/ [10 sep 2014].

19) [OPS-OMS] Organización Panamericana da Saúde, Universidade CES Colombia. 2014. Epidat: programa para análisis epidemiológico de datos. Versión 4.1. España: Consellería de Sanidade, Xunta de Galicia. http://dxsp.sergas.es [15 oct 2014].

20) Pérez-Guerrero L, Milián-Suazo F, Arriaga-Díaz C, Romero-Torres C, Escartín-Chávez M. 2008. Epidemiología molecular de las tuberculosis bovina y humana en una zona endémica de Querétaro, México. Salud pública México, 50(4):286-291.

21) Pérez PF, Ortiz BW, Desmecht $D$, Coral M, Ortiz J, Portaels F, Rigouts $L$, Linder A. 2011. Post-mortem examination and laboratory-based analysis for the diagno- 
sis of bovine tuberculosis among dairy cattle in Ecuador. Preventive Veterinary Medicine, 101:65-72.

22) Pinto PSA, Faria JE, Viloria MIV, Bevilacqua PD, 2002. Examen microbiológico da tuberculose como subsídio à inspeção post-mortem de bovinos. Revista Brasileira de Saúde e Produção Animal, 3:10-15.

23) [SAGARPA] Secretaría de Agricultura, Ganadería, Desarrollo Rural, Pesca y Alimentación. Análisis de los procesos de exportación de ganado bovino en México. 2013. http://www.sagarpa.gob.mx/Paginas/default.aspx [25 jun 2013].

24) [SAGARPA] Secretaría de Agricultura, Ganadería, Desarrollo Rural, Pesca y Alimentación. Prevalencias en Tuberculosis. Cierre enero-diciembre 2014. http:// www.senasica.gob.mx/?id=6246 [25 jun 2015].

25) [SENASICA-SAGARPA] Servicio Nacional de Sanidad, Inocuidad y Calidad Agroalimentaria. 2015. Guía para el seguimiento epidemiológico de la tuberculosis bovina. http://www.senasica.gob.mx/?id=4367 [consulta: 1 abr 2015].

26) [SIAP] Servicio de Información Agroalimentaria y Pesquera. Población Ganadera. http://www.siap.gob.mx/poblacion-ganadera/ [2 de junio 2015].

27) Shitaye JE, Getahun $B$, Alemayehu $T$, Skoric M, Treml F, Fictum P, Vrbas $V$, Pavlik I. 2006. A prevalence study of bovine tuberculosis by using abattoir meat inspection and tuberculin skin testing data, histopathological and IS6110 PCR examination of tissues with tuberculous lesions in cattle in Ethiopia. Veterinární medicina, 51:512-522.

28) Shittu A, Cifton-Hadley RS, Ely ER, Upton PU, Downs SH. 2013. Factors associated with bovine tuberculosis confirmation rates in suspect lesions found in cattle at routine slaughter in Great Britain, 2003-2008. Preventive Veterinary Medicine, 110:395-404.

29) [USDA] United States Department of Agriculture, APHIS. 2009. Veterinary Services Memorandum No. 552.41. Guidelines for Tuberculosis (TB) Reviews in Mexico.

30) Valencia LG, Anda HJ, Lira SE. 1997. Diagnóstico post mortem en decomisos sugestivos a tuberculosis en ganado bovino sacrificado en Baja California. Veterinaria México, 28(3):1-7.

31) Waters RW, Palmer VM, Buddle MB, Vordermeier MH. 2012. Bovine tuberculosis vaccine research: Historical perspectives and recent advances. Vaccine, 30(16):261 1-2622. 